\title{
STOKESIAN DYNAMICS AND THE SETTLING BEHAVIOUR OF PARTICLE-FIBRE-MIXTURES
}

\author{
Dipl.-Math. techn. Markus Feist*, Dipl.-Math. techn. Florian Keller*, \\ Prof. Dr. Willy Dörfler**, Prof. Dr.-Ing. Herman Nirschl*
}

\author{
*Institut für Mechanische Verfahrenstechnik und Mechanik (MVM) \\ **Institut für Angewandte und Numerische Mathematik (IANM) \\ Universität Karlsruhe(TH) \\ 76131 Karlsruhe; Tel: 0721608 2429, Fax: 07216082403
}

\begin{abstract}
Sedimentation of fibres and particles in a fluid can be observed manifold in industrial applications. Not only in waste water treatment but also during the paper recycling process or other separation applications, fibres and particles are involved. The current paper is focussed on the detailed description of the sedimentation behaviour of fibres and particles. Mathematic modelling is used to investigate inter particle influences in detail. In particular a method called Stokesian Dynamics is used to simulate the settling of fibres and particles. The main challenge of the modelling is the dependence of the direction of each fibre on its sedimentation velocity and the different sizes of the particles with a poly-modal particle size distribution. Additionally fibres, particles and the fluid are influencing each other in a significant manner and in a long range. Therefore, one has to take into account the influences of many particles, while calculating the velocity of one particle.
\end{abstract}

Keywords: Mathematic modelling, Stokesian Dynamics, particles and fibres, paper industry, sedimentation, sorting.

\section{INTRODUCTION}

The Sedimentation of organic fibres and inorganic particles can be observed manifold in industrial application. In waste water treatment the water is clarified from solid particles and fibres with the help of sedimentation. Some filtration processes use fibres as an aid to improve the filtration quality. Finally in the paper recycling process one tries to separate the cellulose fibres from the inorganic particles used as filling material or in printing colours. The pure fibres are used again to produce new paper. For all these applications it is necessary to understand the interactions between single particles and the behaviour of the particle collective. In this paper, beside experiments, a mathematical method called Stokesian Dynamics will be introduced. With this method it is possible to investigate in detail the movements of particle collectives with less or many members, without solving partial differential equations.

First analysis on the topic of sedimentation was made by Stokes at the middle of the $19^{\text {th }}$ century (Stokes, 1851). He developed the today well known Stokes formula, describing the sedimentation velocity of a single sphere in a Newtonian fluid. The Stokesian Dynamics method for the simulation of the sedimentation of many particles was introduced (Brady et al. 1988 und Durlofsky et al. 1987) for the first time.

Experiments on the settling behaviour of particles (Beiser 2006, Bickert 1997) show that the settling velocity of the particles depends on the material, the concentration as well as the $\mathrm{pH}$-value of the suspension. In a dilute suspension one gets Stokes sedimentation velocity, which means that the particles settle nearly without interactions. By increasing the concentration clusters are formed that settle faster than single particles. After a further increasing of the concentration, swarm sedimentation can be observed. The particles tend to segregate and the overall velocity is even higher as for the cluster sedimentation. Finally when the concentration becomes too high, the particle interactions slow down the velocity and gets slower than the Stokes-velocity. Separation can often be found in the region of zone sedimentation, and classification in the region of swarm sedimentation. All these experiments were done under the assumption that the particles do not agglomerate during the sedimentation process. A different behaviour can be observed during the sedimentation of fibres. Happel and Brenner (1983) developed an analytical formula for the sedimentation of an ellipsoid. An analytical formula describing the sedimentation velocity of a long 
body, for example a cylinder or a fibre, was given by Batchelor (1970), with the help of the slender body theory. The derivation is based on two solutions, an inner and an outer one, which are coupled in a right manner. The solutions show that the velocity depends on the orientation of the body. This dependence on the orientation is the main difference between particle and fibre sedimentation. Kumar and Ramaro (1991) observed the settling front of a dilute fibre suspension in a cylinder by varying the volume concentration. One sees, that the fibres tend to align in direction of gravity and agglomerate during sedimentation. This phenomenon was discovered using computations by the Stokesian Dynamics method (Bulter und Shaqfeh (2002)) and by experiments from Herzhaft und Guazelli (1998). They tracked single fibres and discovered an alignment and agglomeration.

All described experiments were done with pure fibre or pure particle suspensions. But hardly any experiments can be found with fibre-particle suspensions. Feist et al. (2007) investigates the settling behaviour of screened paper suspensions. They found out that depending on the particle to fibre ratio in the suspension one can observe an alignment which dominates the settling. With a high concentration of fibres, clusters of fibres are formed that sediment very fast and take other and smaller particles with them.

\section{MATHEMATIC MODELLING}

For a better understanding of the inter particle behaviour, the alignment and clustering of the fibres, the mathematical modelling is a necessary tool for the investigations. The simulation will be verified for configurations with a few particles and fibres to investigate the phenomenon between them.

\section{$2.1 \quad$ Theoretical fundamentals}

The fluid can be seen as Newtonian and incompressible. So in general the Navier-Stokes equations

$$
\begin{aligned}
-\frac{\partial \mathrm{v}^{\mathrm{F}}}{\partial \mathrm{t}}+\left(\mathrm{v}^{\mathrm{F}} \cdot \nabla\right) \mathrm{v}^{\mathrm{F}} & =-\nabla \mathrm{p}^{\mathrm{F}}+\frac{1}{\operatorname{Re}} \Delta \mathrm{v}^{\mathrm{F}}+\mathrm{f}, \\
\nabla \cdot \mathrm{v}^{\mathrm{F}} & =0
\end{aligned}
$$

are valid. Because the Reynolds number Re is very small, which is the case for small particles settling in water, the equations (1) and (2) can be simplified to the stationary Stokes equations (3), (4),

$$
\begin{array}{r}
-\Delta \mathrm{v}^{\mathrm{F}}+\nabla \mathrm{p}^{\mathrm{F}}=\mathrm{f}, \\
\nabla \cdot \mathrm{v}^{\mathrm{F}}=0 .
\end{array}
$$

Some special cases excluded, these equations cannot be solved analytically. Stokes (1851) derived the well known analytical formula for the sedimentation velocity of a small settling sphere (5).

$$
\mathrm{v}^{\mathrm{ST}}=\frac{\left(\rho^{\mathrm{S}}-\rho^{\mathrm{F}}\right) \mathrm{a}^{2}}{18 \mu^{\mathrm{F}}} \mathrm{g} .
$$

To calculate the sedimentation of many particles one needs a suitable mathematical model and numerical simulation. The Stokesian Dynamics described by Brady and Bossis (1988), is one possibility. In the following we give a rough description of the method, for a detailed derivation see Brady and Bossis (1988) or Durlofsky et al. (1987).

The basis of this method is the integral representation of the stationary Stokes equations (3), (4). With the help of a multipole expansion it is possible to approximate the velocity disturbance $\mathrm{v}_{\alpha}^{\mathrm{D}}$ of the fluid due to particle $\alpha$, at any position depending on the forces $F_{\alpha}$, torques $T_{\alpha}$.

Besides the influence of the fluid the influences of all the surrounding particles $\beta$ play a vital role on the movements. Denoting $\mathrm{v}^{\prime}\left(\mathrm{z}_{\alpha}\right)=\sum_{\beta \in \text { Partikel } \beta \neq \alpha} \mathrm{v}_{\beta}^{\mathrm{D}}\left(\mathrm{z}_{\alpha}\right)$ by the velocity disturbance generated by all particles except particle $\alpha$ the velocity $\mathrm{v}_{\alpha}$ and angular velocity $\omega_{\alpha}$ of particle $\alpha$ can be expressed in the so called Faxen-Laws (6), (7) 


$$
\begin{aligned}
& \mathrm{v}_{\alpha}=-\frac{\mathrm{F}_{\alpha}}{6 \pi \mu \mathrm{F} a}+\left(1+\frac{1}{6} \mathrm{a}^{2}\right) \mathrm{v}^{\prime}\left(\mathrm{z}_{\alpha}\right), \\
& \omega_{\alpha}=-\frac{\mathrm{T}_{\alpha}}{8 \pi \mu^{\mathrm{F}} \mathrm{a}^{3}}+\frac{1}{2} \nabla \times \mathrm{v}^{\prime}\left(\mathrm{z}_{\alpha}\right) .
\end{aligned}
$$

These laws couple the velocity $v_{\alpha}$ and the angular velocity $\omega_{\alpha}$ of a particle with the force $F_{\alpha}$ and the torque $T_{\alpha}$ of the fluid and disturbance velocity $v^{\prime}\left(z_{\alpha}\right)$. This leads to a system of equations between the velocity und angular velocity of all particles and the forces and torques on them. The matrix $\mathrm{M}$ of the system is called mobility matrix (8).

$$
\left(\begin{array}{c}
\mathrm{v}_{\alpha_{1}} \\
\mathrm{v}_{\alpha_{2}} \\
\vdots \\
\varpi_{\alpha_{1}} \\
\varpi_{\alpha_{2}} \\
\vdots
\end{array}\right)=\mathrm{M}\left(\begin{array}{c}
\mathrm{F}_{\alpha_{1}} \\
\mathrm{~F}_{\alpha_{2}} \\
\vdots \\
\mathrm{T}_{\alpha_{1}} \\
\mathrm{~T}_{\alpha_{2}} \\
\vdots
\end{array}\right)
$$

This system has now to be solved under the knowledge of the velocities in a suitable manner, to get the forces and torques on each single particle. With the help of the Newtonian laws of motion a new velocity and angular velocity is calculated.

This method is derived for spheres of one size so far. Therefore an extension will be presented for different sizes. Finally a fibre is modelled as a chain of spheres.

\subsection{Results}

First, calculations are made with only a few spheres. Eight spheres are placed at the corners of a cube by varying the length of the edges from 3 over 4 to 5 times the particle radius. The result is a periodic sedimentation behaviour. The upper spheres come closer to the lower ones. They overtake each other and finally move to their starting configuration, with exchanged order. Figure 1 shows the z-position at which all particles lay in the same plane for the first time. The calculation shows a good coincidence compared to the results by Durlofsky et al (1987).

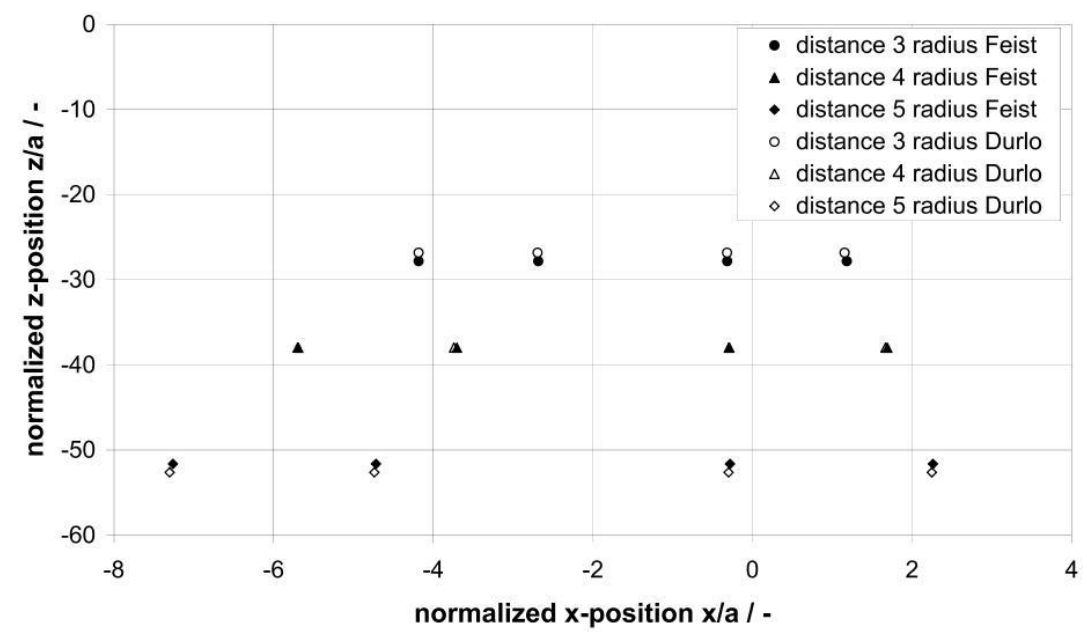

Fig. 1: $\mathrm{x}$-z-projection of the centres of the spheres, which were started in the corners of cubes with an edge length of 3,4 or 5 times the particle radius The positions of the centre of the spheres are shown, when reaching the same plane for the first time. 
Figure 4 shows the result of an experiment, which has no periodic starting position. There is also a good agreement with Durlofsky et al. (1987).

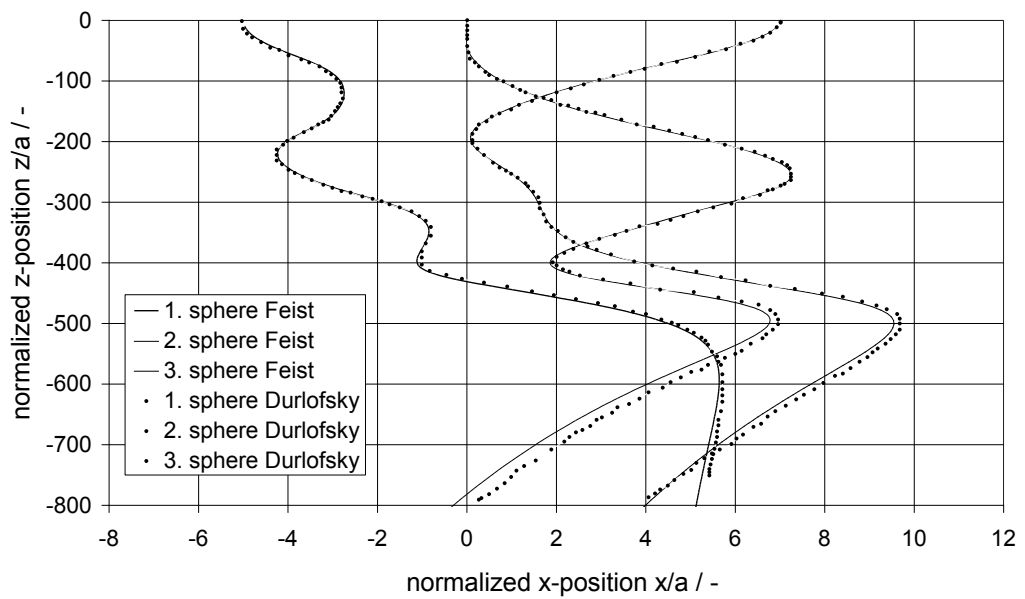

Fig. 2: x-z-projection of the trajectories of the spheres centres of three spheres, started from the positions $(-5,0,0),(0,0,0),(7,0,0)$.

With the next experiment we prove that the modelling of the fibre as a chain of particles can represent the behaviour observed for ellipsoids or fibres. The sedimentation of two fibres with aspect ratio two is compared to the sedimentation of an ellipsoid calculated by Claeys und Brady (1993). One can see that the fibres show the same behaviour (Fig. 3.) as the ellipsoids.

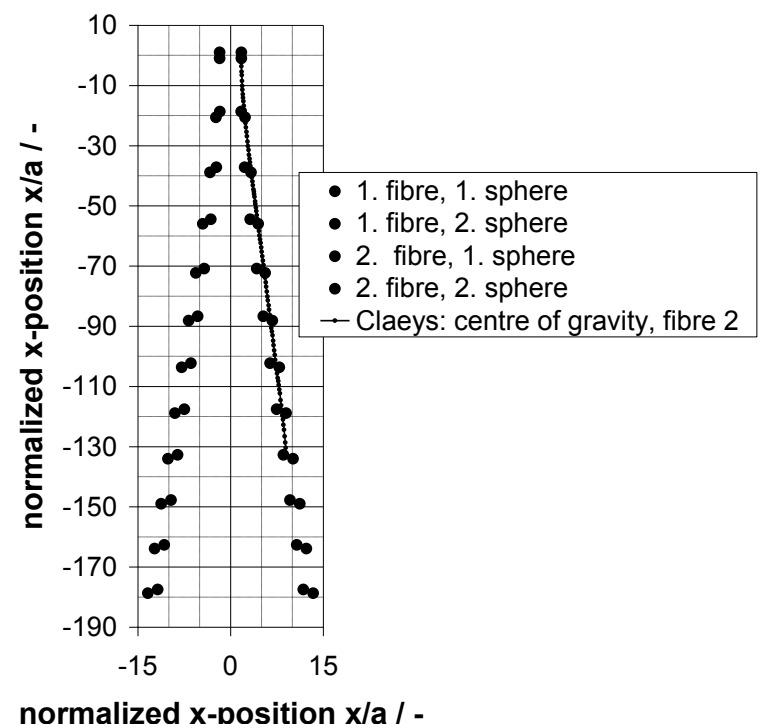

Fig. 3: Sedimentation of two fibres with aspect ratio of two, in comparison to the sedimentation behaviour of an ellipse, simulate by Claeys and Brady (1993). The distance at the beginning is 3.5 times the radius.

\section{CONCLUSION}

A mathematic model has been developed to simulate the sedimentation behaviour of particle-fibre suspensions. The calculations with only a few particles are in good agreement with the calculations which are known from the literature. Additionally the idea to model a fibre as a chain of particles is a suitable possibility to model the sedimentation of fibres. 


\section{REFERENCES}

G. K. Batchelor (1970). The stress system in a suspension of force free particles. J. Fluid Mech., 41:545-570.

M. Beiser (2006). Sedimentation submikroner Partikel in Abhängigkeit physikalisch-chemischer Einflüsse und ihr Separationsverhalten in Dekantierzentrifugen. Dissertation. Universität Karlsruhe (TH).

G. Bickert (1997). Sedimentation feinster suspendierter Partikeln im Zentrifugalfeld. Dissertation. Universität Karlsruhe(TH).

J. F. Brady and G. Bossis (1988). Stokesian Dynamics. Ann. Rev. Fluid Mech, 20:111-157.

J. E. Butler and E. S. G. Shaqfeh (2002). Dynamic simulations of the inhomogeneous sedimentation of rigit fibres. J. Fluid Mech., 468:205-237.

I. L. Claeys and J. F. Brady (1993). Suspensions of prolate spheroids in Stokes flow. Part 1. Dynamics of a finite number of particles in an unbounded fluid. J. Fluid Mech., 251:411-442.

L. Durlofsky, J.F. Brady and G. Bossis (1987). Dynamic simulations of hydrodynamically interacting particles. J. Fluid Mech., 180:21-49.

M. Feist, H. Nirschl, J. Wagner, G. Hirsch and S. Schabel (2007). Experimental results for the settling behaviour of particle-fibre mixtures. Physical Separation in Science and Engineering, vol. 2007, Article ID 91740, 6 pages. doi:10.1155/2007/91740

J. Happel und H. Brenner (1983). Low Reynolds Number Hydrodynamics, Martinus Nijhoff Publishers. The Hague, The Netherlands, 3rd edition.

B. Herzhaft und E. Guazzelli (1999). Experimental study of the sedimentation of dilute and semi--dilute suspensions of fibres. J. Fluid Mech. 384:133-158.

P. Kumar und B.V. Ramarao (1991). Enhancement of the sedimentation rates of fibrous suspensions. Chem Eng. Comm. 108:381-401.

G. G. Stokes (1851). On the Theories of the Internal Friction of Fluids in Motion of Pendulums. Trans. Cambridge Phil. Soc., 9:8-106.

M. Stolarski, C. Eichholz, B. Fuchs and H. Nirschl (2007). Sedimentation acceleration of remanent iron oxide by magnetic flocculation. China Particuology, 3:145-150. 